\title{
Perfil epidemiológico da sífilis congênita no estado de Minas Gerais
}

\author{
Epidemiological profile of congenital syphilis in the state of Minas Gerais \\ Perfil epidemiológico de la sífilis congénita en el estado de Minas Gerais
}

\begin{abstract}
Michelly Martins Nagai ${ }^{1 *}$, Andressa Ferreira Andrade ${ }^{1}$, Débora Braga Soares Bispo ${ }^{1}$, João Carlos Cassimiro', Leilany Marins Andrino ${ }^{1}$, Marcele Soares Côrtes Queiroz ${ }^{1}$, Samara Elisy Miranda Matos $^{1}$, Marisa Costa e Peixoto'.
\end{abstract}

\begin{abstract}
RESUMO
Objetivo: Descrever o perfil epidemiológico da sífilis congênita no estado de Minas de Gerais, com base nos dados do DATASUS no período de 2014 a 2018, a fim de suscitar a busca, prevenção e controle dessa IST. Métodos: Trata-se de um estudo quantitativo, descritivo, transversal. Foram levantados os casos confirmados de Sífilis Congênita dos últimos cinco anos disponíveis no Sistema de Informação de Agravos e Notificação (SINAN - DATASUS), no estado de Minas Gerais. Os dados foram estruturados em tabelas, apresentando distribuição absoluta e relativa das variáveis associadas ao agravo. Resultados: Foi revelado aumento no número absoluto de diagnósticos de sífilis congênita no período em todas as categorias. No entanto, notou-se que os números de diagnósticos realizados durante o parto/curetagem e após o parto diminuíram, com uma redução de 1,3\% e 4,2\% respectivamente, enquanto o número de diagnósticos feitos durante o pré-natal aumentou 5,7\%. Esses dados reforçam o aumento da cobertura e da efetividade do pré-natal nos últimos anos. Conclusão: Observa-se aumento expressivo na incidência da sífilis congênita, que é diagnosticada predominantemente durante o pré-natal. A análise do perfil epidemiológico da sífilis congênita é primordial para o manejo adequado, com intuito de realizar detecção e tratamento precoces evitando complicações.
\end{abstract}

Palavras-chave: Sífilis congênita, Epidemiologia, Cuidado pré-natal.

\begin{abstract}
Objective: To describe the epidemiological profile of congenital syphilis in the state of Minas de Gerais, based on data from DATASUS from 2014 to 2018, in order to encourage search, prevention and control of this STI. Methods: This is a quantitative, descriptive, cross-sectional study. Confirmed cases of Congenital Syphilis in the last five years available in the Notifiable Diseases Information System (SINAN - DATASUS), in the state of Minas Gerais were surveyed. Absolute and relative distribution of the variables associated with the injury was presented in tables. Results: An increase in the absolute number of congenital syphilis diagnoses in the period was revealed in all categories. However, the numbers of diagnoses made during childbirth / curettage and after childbirth decreased, with a reduction of $1.3 \%$ and $4.2 \%$ respectively, while the number of diagnoses made during prenatal care increased by $5,7 \%$. These data reinforce the increase in coverage and effectiveness of prenatal care in recent years. Conclusion: There is a significant increase in the treatment of congenital syphilis, which is predominantly diagnosed during prenatal care. An analysis of the epidemiological profile of congenital syphilis is essential for proper management, aiming at early detection and treatment, avoiding complications.
\end{abstract}

Key words: Congenital syphilis, Epidemiology, Prenatal care.

\section{RESUMEN}

Objetivo: Describir el perfil epidemiológico de la sífilis congénita en Minas de Gerais, a partir de datos de DATASUS de 2014 a 2018, buscando incentivar la prevención y control de esta ITS. Métodos: Estudio cuantitativo, descriptivo, transversal. Se relevaron los casos confirmados de Sífilis Congénita en los últimos cinco años disponibles en el Sistema de Información de Agravamientos de Notificación (SINAN - DATASUS). La distribución absoluta y relativa de las variables asociadas con la enfermedad se presentaron en tablas. Resultados: Hubo un aumento en el número absoluto de diagnósticos de sífilis congénita en todas las categorías. Sin embargo, el número de diagnósticos realizados durante el parto / legrado y después del parto disminuyó, con una reducción del 1,3\% y 4,2\% respectivamente, mientras que el número de diagnósticos realizados durante el prenatal aumentó en un 5, 7\%. Estos datos refuerzan el aumento de la cobertura y la eficacia de la atención prenatal en los últimos años. Conclusión: Existe un aumento significativo en el tratamiento de la sífilis congénita, que se diagnostica predominantemente durante la atención prenatal. El análisis del perfil epidemiológico de la sífilis congénita es fundamental para un adecuado manejo, visando detección y tratamiento precoces y evitando complicaciones.

Palabras clave: Sífilis congénita, Epidemiología, Atención prenatal.

${ }^{1}$ Centro Universitário de Patos de Minas (UNIPAM), Patos de Minas - MG. *E-mail: michellymn@unipam.edu.br 


\section{INTRODUÇÃO}

As infecções sexualmente transmissíveis (IST) são motivo de grandes preocupações para o serviço de saúde pública, uma vez que podem gerar danos à saúde dos indivíduos acometidos. Dentre essas doenças, tem-se a sífilis, uma infecção ocasionada pela bactéria gram-negativa Treponema Pallidum, descoberta em 1905. Segundo Silva LMC, et al. (2019) a infecção é tratável e restrita aos humanos, tendo sua transmissão desencadeada por contato sexual, transfusão sanguínea ou transmissão transplacentária. Além do mais, de acordo com Freitas JLG, et al. (2019), quando essa patologia acomete gestantes pode promover o desenvolvimento da sífilis congênita que, em muitos casos, pode levar ao óbito fetal, restrição de crescimento intrauterino, parto prematuro ou infecção no recém-nascido exposto.

Diante desse cenário de complicações na gestação decorrente da sífilis congênita, com taxa de mortalidade fetal e perinatal elevada ( 40 a 50\%), o Ministério da Saúde instituiu a partir de 1986, pela Portaria Ministerial no 542 de 22/12/1986, a notificação compulsória dos agravos da sífilis congênita no país e, a partir de 2005, a sífilis gestacional também passou a ser notificada compulsoriamente, em virtude da Portaria Ministerial no 33 de 14/07/2005. Além disso, em 2011, por meio da Portaria Ministerial no 104, de 25/01/2011, foi implantada a notificação compulsória da sífilis adquirida. Tais medidas visam priorizar controle e eliminação da transmissão vertical e aumentar o número de nascidos vivos. Assim, evitam-se desfechos negativos ocasionados por falhas nos programas de saúde e pelo tratamento inadequado da gestante e do seu parceiro (MINISTÉRIO DA SAÚDE, 1986; MINISTÉRIO DA SAÚDE, 2005; MINISTÉRIO DA SAÚDE, 2011).

Nesse sentido, vale destacar que se verificou, nos últimos anos, uma elevação dos casos notificados de sífilis congênita, sífilis gestacional e sífilis adquirida. Isso pode ser decorrente do aumento do número de testagem, ocasionado por uma política de distribuição e uso dos testes rápidos (TR), bem como uma diminuição do uso de métodos de barreiras contra as IST, como preservativos (BARBOSA DFR, et al., 2020; BURNS DAR, et al., 2017; LIMA VC, et al., 2017; SOARES KKS, et al., 2020).

A gravidade e as complicações da sífilis congênita dependem do momento da transmissão da bactéria da gestante para o feto, considerando que essa contaminação pode ocorrer intrauterina, perinatal ou pós-natal. A infecção no recém-nascido pode ser sintomática ou assintomática, como ocorre na maior parte dos casos. Entretanto, os primeiros sinais costumam se manifestar nos primeiros dois anos de vida, sendo muito presentes até a quinta semana, quando é possível notar as lesões de pele e mucosas, caracterizando a sífilis congênita precoce (BURNS DAR, et al., 2017; DOMINGUES CSB, et al., 2021).

Por outro lado, quando se observam as manifestações da doença a partir dos dois anos de vida, tem-se a sífilis congênita tardia, na qual há persistência da infecção precoce, além de inflamação cicatricial, podendo evoluir com formação de gomas sifilíticas. Já quando há a forma disseminada, sífilis congênita major, a doença pode se manifestar desde o momento do parto e cursa com uma taxa de até $25 \%$ de mortalidade. Nesse caso, é comum verificar comprometimento difuso da pele e mucosas, com exantema maculopapular e bolhoso, principalmente, em extremidades (pênfigo palmoplantar). Além disso, as alterações sistêmicas da doença levam à formação de hepatomegalia, esplenomegalia, anemia, trombocitopenia, meningoencefalite $\mathrm{e}$, em alguns casos, à pneumonia alba (BURNS DAR, et al., 2017; DOMINGUES CSB, et al., 2021).

De acordo com Ministério da Saúde (2021), o diagnóstico é realizado através do teste rápido (TR) disponível nas redes de atendimento vinculadas ao SUS. Recomenda-se que a gestante seja testada em pelo menos três momentos do pré-natal (primeiro trimestre, terceiro trimestre e no momento do parto ou em episódio de abortamento), de modo que, nos casos de TR positivos, uma nova amostra sanguínea deve ser coletada e realizam-se os testes não treponêmicos Venereal Disease Research Laboratory (VDRL), Rapid Plasma Reagin (RPR) e Toluidine Red Unheated Serum Test (TRUST) para confirmação diagnóstica. Contudo, caso o TR seja reagente, é recomendado o início imediato do tratamento, devido ao risco de transmissão fetal. Desse modo, não é necessário aguardar o resultado do segundo teste (MINISTÉRIO DA SAÚDE, 2021).

Segundo Barbosa DFR, et al. (2020), o tratamento imediato da gestante que tem resultado positivo para sífilis já no primeiro teste é de suma importância, pois devido à rapidez dos TR (média de 30 minutos) há maior facilidade nas condutas de prevenção e no tratamento. Ademais, é necessário realizar anamnese 
clínico-epidemiológica da gestante e exame físico completo do recém-nascido em busca de sinais e sintomas característicos da doença, assim como evidência clínica e/ou laboratorial ocorridas na gestação, parto ou puerpério.

No Protocolo Clínico e Diretrizes Terapêuticas para Prevenção da Transmissão Vertical de HIV, Sífilis e Hepatites Virais (PCDT-TV), lançado em 2019 pelo Ministério da Saúde, o tratamento preconizado e seguro da doença em gestantes é a penicilina benzatina. Outros antibióticos, como as tetraciclinas orais e os macrolídeos, instituídos nos tratamentos da sífilis em adultos não são recomendados devido ao risco de toxicidade ao feto ou por não atravessarem a barreira placentária (MINISTÉRIO DA SAÚDE, 2019).

Contudo, de acordo com o protocolo da OMS, para gestante com sífilis latente recente, em situações específicas, pode-se utilizar ceftriaxona. Ademais, nesses casos, é obrigatório notificar e acompanhar a criança, implementando o tratamento para sífilis congênita, se necessário. O medicamento para esse tipo de acometimento é a benzilpenicilina, de acordo com tratamento prévio ou não da gestante durante a gestação ou de acordo com a titulação do teste não treponêmico da mãe, em comparação com o teste não treponêmico da criança (MINISTÉRIO DA SAÚDE, 2019).

Dessa forma, de acordo Lima VC, et al. (2017), as medidas de prevenção estão intimamente vinculadas aos cuidados com a gestante no período de pré-natal. Devem ser realizadas todas as recomendações preconizadas, como uso correto de preservativos e, quando necessário, tratamento adequado da gestante e de suas parcerias sexuais no momento imediato do diagnóstico da infecção. Além disso, é fundamental conhecer o perfil epidemiológico da doença, visando prevenção, tratamento, controle e redução da mortalidade fetal.

Diante desse contexto da gravidade da sífilis congênita e do aumento dos casos da doença nos últimos anos com elevada morbimortalidade, este estudo tem por objetivo descrever o perfil epidemiológico da sífilis congênita no estado de Minas de Gerais, com base nos dados dos últimos cinco anos completos notificados disponíveis no DATASUS no período de 2014 a 2018, com o intuito de promover a busca de intervenções voltadas à prevenção e controle dessa IST.

\section{MÉTODOS}

Trata-se de um estudo quantitativo, descritivo, transversal. Foram levantados os casos confirmados de Sífilis Congênita dos últimos cinco anos completos disponíveis no SINAN/DATASUS, no estado de Minas Gerais. Foram estudadas as seguintes variáveis: ano de diagnóstico, faixa etária, sexo, raça/cor, classificação final da doença, evolução da doença, faixa etária materna, raça/cor materna, escolaridade materna, realização de pré-natal, momento de diagnóstico da sífilis materna e tratamento do parceiro.

Foram incluídos dados epidemiológicos sobre Sífilis Congênita no estado de Minas Gerais no período de 2014 a 2018 publicados no DATASUS, sendo excluídos os dados da doença não divulgados na base de dados.

Após o levantamento, os dados foram alocados em uma tabela no Microsoft Excel para a estruturação das tabelas apresentadas no estudo, as quais apresentam distribuição absoluta e relativa das variáveis associadas aos casos de Sífilis Congênita. Não foi necessária apreciação desse trabalho pelo Comitê de Ética em Pesquisa (CEP) por se tratar de avaliação de dados públicos.

A pesquisa foi realizada nas seguintes etapas: (1) levantamento bibliográfico, (2) coleta dos dados relacionados à sífilis congênita no SINAN/DATASUS e (3) redação do artigo com a descrição dos dados.

\section{RESULTADOS E DISCUSSÃO}

Ao longo dos cinco anos, no período de 2014 a 2018, foram notificados 8.196 casos de sífilis congênita no estado de Minas Gerais. Durante esse período, a maioria das notificações ocorreu no ano de 2018 com 2.365 casos, correspondendo a $28,8 \%$, seguido por $2017 \mathrm{com} 22,9 \%$ do total de casos. Identificou-se aumento no número de casos a cada ano, apresentando em 2018 um número de casos 2,4 vezes maior que o ano de 2014 (Tabela 1). 
Tabela 1 - Distribuição absoluta (n) e percentual (\%) dos casos de sífilis congênita por ano de diagnóstico. Minas Gerais, 2014 a 2018.

\begin{tabular}{lc}
\hline Ano de diagnóstico & Casos confirmados n $(\%)$ \\
\hline 2014 & $968(11,8)$ \\
2015 & $1459(17,8)$ \\
2016 & $1527(18,6)$ \\
2017 & $1877(22,9)$ \\
2018 & $2365(28,9)$ \\
\hline Total & $\mathbf{8 1 9 6 ( 1 0 0 )}$ \\
\hline
\end{tabular}

Nota: Excluídos casos não residentes no Brasil, maiores de 12 anos de idade, notificações descartadas ou sem investigação. Fonte: Nagai MM, et al., 2021; dados extraídos de Ministério da Saúde/SVS - Sistema de Informação de Agravos de Notificação - Sinan Net, 2021.

Outros estudos mostram resultados semelhantes. Uma pesquisa realizada entre 2007 a 2016 no estado do Pará evidenciou aumento expressivo de casos de sífilis congênita, indicando crescimento de 2,7 vezes no número de notificações (SILVA LMC, et al., 2019). Outro estudo realizado na Colômbia evidenciou aumento de 1,5 vezes no número de casos entre 2005 e 2011 (TEIXEIRA LO, et al., 2018). Nos Estados Unidos, a prevalência de sífilis congênita aumentou 27,5\% no período de 2013 a 2014 (ANDRADE ALMB, et al.,2018). No Brasil, no período de 2010 a 2017 houve aumento de 3 vezes no número de casos notificados, fato possivelmente relacionado à diminuição do uso de preservativos, difusão do uso de testes rápidos na Atenção Primária, ampliação das notificações e desabastecimento mundial de penicilina (MINISTÉRIO DA SAÚDE, 2018).

A sífilis congênita é considerada problema de saúde pública pela OMS. Em 2010, foi estabelecido uma meta de redução dos casos dessa patologia na América Latina pelo Fundo das Nações Unidas para a Infância (UNICEF) e pela OPAS (ANDRADE ALMB, et al., 2018). No entanto, o resultado desse estudo demonstra o insucesso dessa meta, uma vez que o número de casos cresce constantemente.

Durante os anos de 2014 a 2018, indivíduos com idade de até seis dias apresentaram maiores taxas de sífilis congênita, com o percentual médio de $95,9 \%$, variando de $94,6 \%$ a $96,7 \%$ ao longo do período analisado. A segunda faixa etária mais acometida foi a de indivíduos com idade igual ou superior a 28 dias e inferior a um ano, responsáveis por $1,8 \%$ de todos os casos ao longo dos anos (Tabela 2). Este achado assemelha-se ao de uma pesquisa realizada entre 2009 a 2018 no estado do Acre, que mostrou 96,2\% dos diagnósticos de sífilis congênita ocorrendo em até seis dias de vida (BRANCO TJT, et al., 2020).

Em relação ao sexo dos indivíduos diagnosticados com sífilis congênita no período estudado, observaramse proporções semelhantes entre sexos feminino e masculino. Foram notificados 3915 casos da doença em indivíduos do sexo feminino, equivalentes a $47,8 \%$ do total, e 3778 casos em indivíduos do sexo masculino, representando $46,1 \%$ de todas as notificações (Tabela 2). Branco TJT, et al. (2020) observaram resultados similares em estudo realizado no estado do Acre no qual $48,8 \%$ dos casos notificados da doença correspondiam ao sexo masculino e $46,6 \%$ ao sexo feminino. Uma vez que a infecção ocorre por via transplacentária e não tem predileção por nenhum dos sexos (SILVA LCVG, et al., 2017), pode-se esperar os resultados encontrados.

No que diz respeito à raça/cor, a maioria dos casos notificados de sífilis congênita era de indivíduos pardos. Foram registrados 4015 casos de indivíduos pardos que, somados aos números de casos de indivíduos pretos, amarelos e indígenas, correspondem a 55,4\% dos casos. É importante ressaltar que em 2014 não havia a informação sobre a variável (dado ignorado ou em branco), o que poderia aumentar ainda mais tal proporção (Tabela 2). Esse número pode ser reflexo de características da população brasileira constituída por proporção elevada de indivíduos que se autodeclaram como pardos (INSTITUTO BRASILEIRO DE GEOGRAFIA E ESTATÍSTICA (IBGE), 2010). Corroborando os resultados encontrados, outros estudos mostram predomínio de sífilis congênita em indivíduos não brancos em diversos estados do país onde essa parcela da população também é majoritária, tal como ocorre em Minas Gerais (BRANCO TJT, et al., 2020; FREITAS JLG, et al., 2019; MOREIRA KFA, et al., 2017).

Quanto à classificação final da sífilis congênita apresentada neste estudo, 7736 (94,4\%) casos foram classificados como sífilis congênita recente. Observa-se também que o percentual de natimorto/aborto por sífilis aumentou durante o período estudado, passando de 9,3\% em 2014 para 25,7\% em 2018 (Tabela 2). 
Similarmente, trabalhos realizados em Rondônia e Pará mostraram que a maior parte dos casos notificados de sífilis congênita, $82,6 \%$ e $93 \%$ respectivamente, são classificados como sífilis congênita recente (FREITAS JLG, et al., 2019; SILVA LMC, et al., 2019).

É importante destacar que o grande número de diagnósticos precoces de sífilis congênita realizados permite acompanhamento e tratamento adequados da criança, evitando consequências na vida adulta por prevenir danos ao sistema nervoso central, ossos, articulações, dentes, olhos e pele, incluindo a tríade de Hutchinson: ceratite intersticial, surdez por lesão do oitavo par de nervos cranianos e dentes de Hutchinson (COOPER JM, et al., 2016). Não se deve negligenciar, entretanto, o aumento da proporção dos casos de natimorto/aborto pela doença que, muitas vezes, podem estar relacionados à má assistência pré-natal e ao tratamento inadequado das gestantes portadoras de sífilis (SARACENI V, et al., 2017).

Considerando a evolução dos casos notificados, nota-se que $92,5 \%$ permaneceram vivos (Tabela 2). No estado do Acre, Branco TJT, et al. (2020) encontraram resultado semelhante com 93\% dos casos notificados permanecendo vivos. Tais achados podem estar relacionados às ações de saúde que vêm sendo implementadas nos últimos anos para melhorar o preparo das equipes de saúde não só para tratar, como também para prevenir e erradicar a sífilis congênita (MINISTÉRIO DA SAÚDE, 2019). Desde 2016, o Brasil em parceria com a OPAS e a OMS vem intensificando ações para prevenir e tratar a sífilis congênita, com o intuito de eliminá-la até 2030 (MINISTÉRIO DA SAÚDE, 2018).

Tabela 2 - Distribuição absoluta (n) e percentual (\%) dos casos de sífilis congênita segundo os dados sociodemográficos, classificação final e evolução da doença por ano de diagnóstico. Minas Gerais, 2014 a 2018.

\begin{tabular}{|c|c|c|c|c|c|c|}
\hline \multirow[b]{2}{*}{ Variável } & \multicolumn{6}{|c|}{ Ano } \\
\hline & $\begin{array}{l}2014 \\
\text { n (\%) }\end{array}$ & $\begin{array}{l}2015 \\
\text { n (\%) }\end{array}$ & $\begin{array}{l}2016 \\
\text { n (\%) }\end{array}$ & $\begin{array}{l}2017 \\
\text { n (\%) }\end{array}$ & $\begin{array}{l}2018 \\
\text { n (\%) }\end{array}$ & $\begin{array}{l}\text { Total } \\
\text { n (\%) }\end{array}$ \\
\hline \multicolumn{7}{|l|}{ Faixa Etária } \\
\hline $\begin{array}{l}\text { até } 6 \text { dias } \\
7-27 \text { dias } \\
28 \text { dias a }<1 \text { ano } \\
1 \text { ano ( } 12 \text { a } 23 \text { meses) } \\
2 \text { a } 4 \text { anos } \\
5 \text { a } 12 \text { anos }\end{array}$ & $\begin{array}{c}916(94,6) \\
26(2,7) \\
21(2,2) \\
5(0,5) \\
-(-) \\
-(-) \\
\end{array}$ & $\begin{array}{c}1388(95,1) \\
30(2,1) \\
35(2,4) \\
3(0,2) \\
2(0,1) \\
1(0,1)\end{array}$ & $\begin{array}{c}1471(96,3) \\
17(1) \\
27(1,8) \\
7(0,5) \\
4(0,3) \\
1(0,1) \\
\end{array}$ & $\begin{array}{c}1798(95,7) \\
30(1,6) \\
40(2,1) \\
5(0,3) \\
3(0,2) \\
1(0,1) \\
\end{array}$ & $\begin{array}{c}2288(96,7) \\
40(1,7) \\
31(1,3) \\
3(0,1) \\
1(0) \\
2(0) \\
\end{array}$ & $\begin{array}{c}7861(95,9) \\
143(1,7) \\
154(1,9) \\
23(0,3) \\
10(0,1) \\
5(0,1)\end{array}$ \\
\hline Total & $968(100)$ & $1459(100)$ & $1527(100)$ & $1877(100)$ & $2365(100)$ & $8196(100)$ \\
\hline \multicolumn{7}{|l|}{ Sexo } \\
\hline $\begin{array}{l}\text { Ignorado } \\
\text { Masculino } \\
\text { Feminino }\end{array}$ & $\begin{array}{r}45(4,6) \\
452(46,7) \\
471(48,7)\end{array}$ & $\begin{array}{c}68(4,7) \\
710(48,7) \\
681(46,6)\end{array}$ & $\begin{array}{c}83(5,5) \\
697(45,6) \\
747(48,9)\end{array}$ & $\begin{array}{c}150(8) \\
834(44,4) \\
893(47,6)\end{array}$ & $\begin{array}{c}157(6,6) \\
1085(45,9) \\
1123(47,5)\end{array}$ & $\begin{array}{c}503(6,1) \\
3778(46,1) \\
3915(47,8)\end{array}$ \\
\hline Total & $968(100)$ & $1459(100)$ & $1527(100)$ & $1877(100)$ & $2365(100)$ & $8196(100)$ \\
\hline \multicolumn{7}{|l|}{ Raça/cor } \\
\hline Ignorado/Branco & $318(32,9)$ & $490(33,6)$ & $487(31,9)$ & $366(19,5)$ & $353(14,9)$ & $2014(24,6)$ \\
\hline Branca & $175(18,1)$ & $259(17,8)$ & $262(17,2)$ & $401(21,4)$ & $546(23,1)$ & $1643(20)$ \\
\hline Preta & $72(7,4)$ & $80(5,4)$ & $90(5,9)$ & $104(5,5)$ & $139(5,9)$ & $485(5,9)$ \\
\hline Amarela & $4(0,4)$ & $2(0,1)$ & $7(0,4)^{\prime}$ & $8(0,4)$ & $12(0,5)^{\prime}$ & $33(0,4)^{\prime}$ \\
\hline Parda & $399(41,2)$ & $627(43)$ & $680(44,5)$ & $997(53,1)$ & $1312(55,5)$ & $4015(49)$ \\
\hline Indígena & $-(-)$ & $1(0,1)$ & $1(0,1)$ & $1(0,1)$ & $3(0,1)$ & $6(0,1)$ \\
\hline Total & $968(100)$ & $1459(100)$ & $1527(100)$ & $1877(100)$ & $2365(100)$ & $8196(100)$ \\
\hline \multicolumn{7}{|l|}{ Classificação final } \\
\hline SC recente & $922(95,2)$ & $1383(94,8)$ & $1435(94)$ & $1733(92,4)$ & $2263(95,7)$ & $7736(94,4)$ \\
\hline SC tardia & $-(-)$ & $4(0,3)$ & $5(0,3)$ & $4(0,2)$ & $3(0,1)$ & $16(0,2)$ \\
\hline $\begin{array}{l}\text { Natimorto/Aborto por sífilis } \\
\text { Descartado }\end{array}$ & $\begin{array}{c}17(1,8) \\
29(3)\end{array}$ & $\begin{array}{l}24(1,6) \\
48(3,3)\end{array}$ & $25\left(\begin{array}{l}1,6 \\
4,1\end{array}\right)$ & $70(3,7)$ & $47(2)$ & $183(2,2)$ \\
\hline Total & $968(100)$ & $\frac{40(10)}{1459(100)}$ & $\frac{0<(4,1)}{1527(100)}$ & $\frac{10(3,1)}{1877(100)}$ & $\frac{5<(<,<)}{2365(100)}$ & $\frac{201(3, \angle)}{8196(100)}$ \\
\hline \multicolumn{7}{|l|}{ Evolução } \\
\hline Tgnorado/Branco & $91(9,9)$ & $80(5,8)$ & $44(3)$ & $49(2,8)$ & $74(3,3)$ & $338(4,4)$ \\
\hline Vivo & $806(87,4)$ & $1254(90,4)$ & $1356(94,2)$ & $1629(93,8)$ & $2128(93,9)$ & $7173(92,5)$ \\
\hline Obito pelo agravo notificado & $20(2,2)$ & $34(2,4)$ & $31(2,2)$ & $40(2,3)$ & $51(2,2)$ & $176(2,3)$ \\
\hline Obito por outra causa & $5(0,5)$ & $19(1,4)$ & $9(0,6)$ & $19(1,1)$ & $13(0,6)$ & $65(0,8)$ \\
\hline Total & $922(100)$ & $1387(100)$ & $1440(100)$ & $1737(100)$ & $2266(100)$ & $7752(100)$ \\
\hline
\end{tabular}

Notas: Excluídos casos não residentes no Brasil, maiores de 12 anos de idade, notificações descartadas ou sem investigação. SC: Sífilis Congênita. Fonte: Nagai MM, et al., 2021; dados extraídos de Ministério da Saúde/SVS - Sistema de Informação de Agravos de Notificação - Sinan Net, 2021.

Quanto à faixa etária materna, observou-se maior acometimento de gestantes com idade entre 20 e 29 anos $(52,2 \%)$ e de gestantes com idade entre 15 e 19 anos $(24,3 \%)$, enquanto as gestantes menos acometidas foram aquelas com 40 anos ou mais (1,8\%) e 10 a 14 anos $(0,8 \%)$ (Tabela 3). Esses resultados foram similares aos do estudo de Branco TJT, et al. (2020) na região norte do país. Outros estudos brasileiros também registraram a maioria das gestantes acometidas na faixa etária de 20 a 29 anos (MASCHIO-LIMA T, et a., 2019; MOREIRA KFA, et al., 2017). De acordo com a pesquisa de Gray PB, et al. (2019), a frequência 
de relações sexuais de adultos solteiros é maior entre menores de 39 anos, aumentando o número de gestações e o risco de IST nessa faixa etária. Esse achado poderia estar relacionado à maior taxa de sífilis congênita observada em mulheres jovens encontrada no presente estudo e nos demais estudos citados.

Com relação à raça/cor materna, a maioria das gestantes se autodeclarou como parda $(54,8 \%)$, seguidas de mulheres que se autodeclararam como brancas (18,2\%), pretas $(13,7 \%)$, raça/ cor ignorada $(12,6 \%)$, amarelas $(0,6 \%)$ e, por último, indígenas $(0,1 \%)$ (Tabela 3). O estudo de Lafetá KRG, et al. (2016), sobre a sífilis congênita e materna em um município de Minas Gerais, também encontrou que a maioria das gestantes se autodeclarava como pardas $(63,4 \%)$ e brancas $(16,1 \%)$, enquanto $7,5 \%$ das mulheres se consideravam pretas, $1,1 \%$ amarelas e em $11,9 \%$ dos casos não havia essa informação.

Ainda em relação à raça/cor materna, resultados análogos também foram encontrados nas pesquisas de Moreira KFA, et al. (2017) e Freitas JLG, et al. (2019), nas quais a maioria das gestantes com sífilis congênita eram pardas, brancas e pretas. Outros estudos dividiram as mulheres em pardas e não pardas ou brancas e não brancas, sendo a maioria considerada parda e não branca, respectivamente (SILVA LMC, et al., 2019; SILVA MJN, et al., 2020). Esses resultados estão de acordo com a população brasileira que se autodeclara majoritariamente como parda, seguida de branca, preta, amarela e indígena (IBGE, 2019). O fato de muitos responderem com ignorado/não informado ou a generalização em parda ou não parda, branca ou não branca dificulta a análise da incidência da doença nas diferentes raças/etnias da população brasileira.

Tabela 3 - Distribuição absoluta (n) e percentual (\%) dos casos de sífilis congênita segundo dados sociodemográfico maternos, realização de pré-natal e momento de diagnóstico materno e tratamento do parceiro. Minas Gerais, 2014 a 2018.

\begin{tabular}{|c|c|c|c|c|c|c|}
\hline \multirow{3}{*}{ Variável } & \multicolumn{6}{|c|}{ Ano } \\
\hline & 2014 & 2015 & 2016 & 2017 & 2018 & Total \\
\hline & n (\%) & n (\%) & n (\%) & n (\%) & n (\%) & n (\%) \\
\hline \multicolumn{7}{|l|}{ Faixa etária materna ${ }^{a}$} \\
\hline 10 a 14 anos & $4(0,4)$ & $16(1,1)$ & $16(1,1)$ & $11(0,6)$ & $14(0,6)$ & $61(0,8)$ \\
\hline 15 a 19 anos & $216(22,8)$ & $347(24,4)$ & $353(24)$ & $476(26.2)$ & $575(23,6)$ & $1967(24,3)$ \\
\hline 20 a 29 anos & $491(51,8)$ & $693(48,7)$ & $773(52,5)$ & $944(52)$ & $1333(54,4)$ & $4234(52,2)$ \\
\hline 30 a 39 anos & $208(22)$ & $301(21,2)$ & $277(18,8)$ & $323(17,8)$ & $434(17,7)$ & $1543(19)$ \\
\hline 40 anos ou mais & $16(1,7)^{\prime}$ & $27(1,9)^{\prime}$ & $32(2,1)$ & $27(1,5)$ & $47(1,9)$ & $149(1,8)$ \\
\hline Ignorado & $12(1,3)$ & $38(2,7)$ & $22(1,5)$ & $34(1,9)$ & $46(1,8)$ & $152(1,9)$ \\
\hline Total & $947(100)$ & $1422(100)$ & $1473(100)$ & $1815(100)$ & $2449(100)$ & $8106(100)$ \\
\hline \multicolumn{7}{|l|}{ Raça/cor materna ${ }^{a}$} \\
\hline Branca & $163(17,2)$ & $263(18,5)$ & $261(17,7)$ & $333(18,3)$ & $458(18,7)$ & $1478(18,2)$ \\
\hline Preta & $154(16,3)$ & $193(13,6)$ & $182(12,4)$ & $246(13,6)$ & $328(13,4)$ & $1103(13,7)$ \\
\hline Amarela & $4(0,4)$ & $12(0,8)$ & $6(0,4)$ & $10(0,5)$ & $17(0,8)$ & $49(0,6)$ \\
\hline Parda & $471(49,7)$ & $731(51,4)$ & $816(55,4)$ & $1027(56,6)$ & $1394(56,9)$ & $4439(54,8)$ \\
\hline Indígena & $3(0,3)$ & $3(0,2)$ & $2(0,1)$ & $2(0,1)$ & $2(0)$ & $12(0,1)$ \\
\hline Ignorada & $152(16,1)$ & $220(15,5)$ & $206(14)$ & $197(10,9)$ & $250(10,2)$ & $1025(12,6)$ \\
\hline Total & $947(100)$ & $1422(100)$ & $1473(100)$ & $1815(100)$ & $2449(100)$ & $8106(100)$ \\
\hline \multicolumn{7}{|l|}{ Escolaridade materna } \\
\hline Tgnorado/Branco & $462(47,8)$ & $563(38,6)$ & $644(42,2)$ & $742(39,6)$ & $860(36,4)$ & $3271(39,9)$ \\
\hline Analfabeta & $6(0,6)$ & $4(0,3)$ & $8(0,5)$ & $10(0,5)$ & $16(0,7)$ & $44(0,5)$ \\
\hline Ens Fund incompleto & $213(22)$ & $325(22,2)$ & $309(20,2)$ & $391(20,8)$ & $482(20,4)$ & $1720(21)$ \\
\hline Ens Fund completo & $86(8,9)$ & $178(12,2)$ & $192(12,6)$ & $199(10,6)$ & $257(10,9)$ & $912(11,1)$ \\
\hline En Médio incompleto & $89(9,2)$ & $146(10)^{\prime}$ & $145(9,5)^{\prime}$ & $225(12)$ & $292(12,3)$ & $897(10,9)$ \\
\hline Ens Médio completo & $89(9,2)$ & $214(14,7)$ & $202(13,3)$ & $264(14,1)$ & $395(16,7)$ & $1164(14,2)$ \\
\hline Ens Superior incompleto & $9(0,9)^{\prime}$ & $14(1)$ & $8(0,5)$ & $15(0,8)$ & $29(1,2)$ & $75(0,9)$ \\
\hline Ens Superior completo & $6(0,6)$ & $5(0,3)$ & $13(0,8)$ & $10(0,5)$ & $20(0,8)$ & $54(0,7)$ \\
\hline Não se aplica & $8(0,8)$ & $10(0,7)$ & $6(0,4)$ & $21(1,1)$ & $14(0,6)$ & $59(0,8)$ \\
\hline Total & $968(100)$ & $1459(100)$ & $1527(100)$ & $1877(100)$ & $2365(100)$ & $8196(100)$ \\
\hline \multicolumn{7}{|l|}{ Realizou pré-natal } \\
\hline Tgnorado/Branco & $28(3)$ & $77(5,3)$ & $65(4,2)$ & $42(2,2)$ & $52(2,2)$ & $264(3,2)$ \\
\hline Sim & $804(83)$ & $1233(84,5)$ & $1319(86,4)$ & $1654(88,1)$ & $2111(89,3)$ & $7121(86,9)$ \\
\hline Não & $136(14)$ & $149(10,2)$ & $143(9,4)$ & $181(9,6)$ & $202(8,5)$ & $811(9,9)$ \\
\hline Total & $968(100)$ & $1459(100)$ & $1527(100)$ & $1877(100)$ & $2365(100)$ & $8196(100)$ \\
\hline \multicolumn{7}{|l|}{ Diagnóstico de SM } \\
\hline Ignorado/Branco & $27(2,8)$ & $77(5,3)$ & $49(3,2)$ & $50(2,7)$ & $53(2,2)$ & $256(3,1)$ \\
\hline Durante o pré-natal & $597(61,7)$ & $891(61,1)$ & $985(64,5)$ & $1203(64,1)$ & $1594(67,4)$ & $5270(64,3)$ \\
\hline No parto/curetagem & $242(25)$ & $378(25,9)$ & $389(25,5)$ & $501(26,7)$ & $561(23,7)$ & $2071(25,3)$ \\
\hline Após o parto & $98(10,1)$ & $105(7,2)$ & $94(6,2)$ & $107(5,7)^{\prime}$ & $139(5,9)$ & $543(6,6)$ \\
\hline Não realizado & $4(0,4)$ & $8(0,5)$ & $10(0,6)$ & $16(0,8)$ & $18(0,8)$ & $56(0,7)$ \\
\hline Total & $968(100)$ & $1459(100)$ & $1527(100)$ & $1877(100)$ & $2365(100)$ & $8196(100)$ \\
\hline \multicolumn{7}{|l|}{ Trat do parceiro } \\
\hline Ignorado/Branco & $276(28,5)$ & $337(23,1)$ & $283(18,5)$ & $271(14,4)$ & $334(14,1)$ & $1501(18,3)$ \\
\hline & $120(12,4)$ & $232(15,9)$ & $272(17,8)$ & $377(20,1)$ & $511(21,6)$ & $1512(18,4)$ \\
\hline Não & $572(59,1)$ & $890(61)$ & $972(63,7)$ & $1229(65,5)$ & $1520(64,3)$ & $5183(63,3)$ \\
\hline Total & $968(100)$ & $1459(100)$ & $1527(100)$ & $1877(100)$ & $2365(100)$ & $8196(100)$ \\
\hline
\end{tabular}

Notas: Excluídos casos não residentes no Brasil, maiores de 12 anos de idade, notificações descartadas ou sem investigação. Ens: ensino; Fund: fundamental; SM: sífilis materna; Trat: tratamento. ${ }^{2}$ Dados preliminares para os últimos cinco anos. Fonte: Nagai MM, et al., 2021; dados extraídos de Ministério da Saúde/SVS Sistema de Informação de Agravos de Notificação - Sinan Net e Departamento de Doenças de Condições Crônicas e Infecções Sexualmente Transmissíveis, 2021. 
Em relação à escolaridade materna, durante o período analisado foram mais frequentes casos em que esta variável não foi especificada, correspondendo a $39,9 \%$ dos casos. A segunda categoria mais prevalente foi ensino fundamental incompleto, representando $21 \%$ dos casos (Tabela 3). Dessa forma, é imprescindível observar que a baixa escolaridade é um fator limitante ao acesso às informações relacionadas à conscientização sobre saúde sexual e medidas de prevenção da sífilis, além de destacar a importância do conhecimento sobre o autocuidado (SILVA LMC, et al., 2019). Sendo assim, é possível considerar que a restrição do conhecimento sobre práticas de saúde é um fator de risco para a sífilis e evolução para sífilis congênita em mulheres com baixa escolaridade, em razão da maior vulnerabilidade à contaminação com IST dessas mulheres (BRANCO TJT, et al., 2020).

No que tange à realização do pré-natal, pode-se notar que durante o período analisado foram mais frequentes casos de gestantes que realizaram o pré-natal, representando $86,9 \%$ dos casos (Tabela 3). Apesar de a realização do pré-natal não favorecer a transmissão vertical da sífilis por meio do seu controle, observamse falhas neste atendimento, sobretudo no diagnóstico e também no tratamento, que podem comprometer o controle dessa transmissão (SOARES KKS, et al., 2020).

Vale ressaltar, nesse sentido, que não é suficiente a realização do pré-natal sem que exista qualidade. Uma vez que o acompanhamento pré-natal inadequado configura fator de risco para a sífilis congênita, é válido destacar que os maiores problemas observados são: anamnese incompleta ou inadequada, não realização de sorologia para sífilis nos períodos preconizados ( $1^{\circ}$ e $3^{\circ}$ trimestres), interpretação inadequada da sorologia, além de falha no reconhecimento dos sinais de sífilis materna (TOLDO MKS, et al., 2018). Ademais, a falta de tratamento do parceiro sexual e a falta de interdisciplinaridade entre as equipes obstétrica e pediátrica também devem ser evitadas cuidadosamente (DANTAS ASC, et al., 2020).

Em referência à sífilis materna, os resultados encontrados mostraram aumento no número absoluto de diagnósticos de sífilis congênita entre os anos de 2014 e 2018 em todas as categorias. Entretanto, observouse que, em porcentagem, os números de diagnósticos realizados durante o parto/curetagem e após o parto diminuíram, com uma redução de $1,3 \%$ e $4,2 \%$ respectivamente, enquanto o número de diagnósticos feitos durante o pré-natal aumentou $5,7 \%$ e foi a categoria que mais cresceu. $O$ número médio de diagnósticos da doença durante o pré-natal foi de $63,8 \%$ no período (Tabela 3). Um estudo realizado para analisar o perfil epidemiológico da sífilis congênita em um município do nordeste brasileiro encontrou resultado semelhante com $62,1 \%$ das gestantes recebendo o diagnóstico da doença durante o pré-natal (LIMA VC, et al., 2017).

É importante enfatizar que o aumento do número de diagnósticos de sífilis congênita durante o pré-natal pode estar relacionado ao aumento da cobertura e da efetividade do acompanhamento pré-natal nos últimos anos (MINISTÉRIO DA SAÚDE, 2012). Além disso, o aumento no número de casos de sífilis materna no Brasil pode ser explicado pela maior cobertura de testagem e amplificação dos testes rápidos feitos nas Unidades Básicas de Saúde (MOTTA IA, et al., 2018).

É fundamental destacar que o diagnóstico tardio da sífilis materna quase duplica o risco de ocorrência de sífilis congênita e suas complicações (FREITAS JLG, et al., 2019). Assim, a permanência do diagnóstico tardio realizado durante o parto/curetagem ou após o parto bem como o número de casos de diagnósticos não realizados no presente estudo reforçam a necessidade da implementação de políticas públicas visando a qualificação dos profissionais de saúde. Tais políticas são imprescindíveis para que além da notificação, o tratamento da sífilis materna ocorra de forma adequada durante o pré-natal contribuindo para a saúde materna e para evitar a ocorrência de sífilis congênita (CONCEIÇÃO HN, et al., 2019).

No tocante ao tratamento do parceiro, observa-se que apesar de os casos tratados terem aumentado durante o período estudado, passando de 12,4\% em 2014 para 21,6\% em 2018, o número de casos não tratados foi consideravelmente maior que o de casos tratados em todos os anos e também houve um aumento desses casos não tratados que passaram de 59,1\% em 2014 para 64,3\% em 2018 (Tabela 3). Em pesquisa realizada com casos notificados de sífilis congênita no Brasil, no Rio Grande do Sul e no município de Pelotas entre 2006 e 2015, Holztrattner JS, et al. (2019) observaram resultados símiles com o percentual de parceiros tratados não atingindo $20,5 \%$ em nenhuma das três esferas. 
Algumas questões são levantadas para explicar o não tratamento do parceiro. Muitas vezes, associa-se aos homens menor preocupação com sua saúde e autocuidado. Ademais, ainda é muito presente no Brasil a ideia de que saúde pública é restrita a crianças, mulheres e idosos, sugerindo também menor presença dos homens nos serviços de saúde (MAGALHÃES DMS, et al., 2013).

A inclusão do parceiro no pré-natal tem sido estratégia importante para a abordagem do problema da sífilis e é determinante para a cura eficaz da mãe e, consequentemente, evitar a sífilis congênita (MAGALHÃES DMS, et al., 2013). A sorologia do parceiro é fundamental para prevenir a infecção e a transmissão para o feto (COOPER JM, et al., 2016). Além disso, para que o tratamento da sífilis materna seja considerado efetivo, o tratamento do parceiro, caso seja teste positivo, deve ser feito simultaneamente ao da gestante (CONCEIÇÃO HN, et al., 2019).

\section{CONCLUSÃO}

Nota-se crescimento significativo na incidência da sífilis congênita no decorrer do tempo no estado de Minas Gerais. A doença vem acometendo principalmente indivíduos pardos, sendo classificada como sífilis congênita recente e a maioria dos infectados sobrevive à doença. A sífilis materna afeta primordialmente mulheres jovens, pardas, com ensino fundamental incompleto, que passam por assistência pré-natal e recebem o diagnóstico durante esse acompanhamento. A maioria dos parceiros não tem acesso ao tratamento. É fundamental enfatizar a necessidade do preenchimento correto das fichas de notificação da doença. Tal ação permite analisar os dados epidemiológicos da sífilis congênita, fornecendo subsídios para o manejo adequado. Dessa forma, pode-se realizar detecção e tratamento precoces evitando complicações, bem como promover ações de educação em saúde sobre as formas de transmissão da infecção direcionadas à parcela da população sob maior risco.

\section{REFERÊNCIAS}

1. ANDRADE ALMB, et al. Diagnóstico tardio de sífilis congênita: uma realidade na atenção à saúde da mulher e da criança no Brasil. Revista Paulista de Pediatria, 2018; 36(3): 376-381.

2. BARBOSA DFR, et al. Perfil epidemiológico da sífilis congênita em gestantes no município de Maceió. Revista Eletrônica Acervo Saúde, 2020; 12(11): 1-9.

3. BRANCO TJT, et al. Perfil epidemiológico dos casos notificados de sífilis congênita no estado do Acre nos anos de 2009-2018. Revista Eletrônica Acervo Saúde, 2020; 12(9): 1-10.

4. BURNS DAR, et al. Tratado de Pediatria: Sociedade Brasileira de Pediatria. 4 ed. Barueri, SP: Manole, $2017 ; 2564$ p.

5. CONCEIÇÃO HN, et al. Análise epidemiológica e espacial dos casos de sífilis gestacional e congênita. Revista Saúde em Debate, 2019; 43 (123): 1145-1158.

6. COOPER JM, et al. Em tempo: a persistência da sífilis congênita no Brasil - Mais avanços são necessários! Revista Paulista de Pediatria, 2016; 34(3): 251-253.

7. DANTAS ASC, et al. As diversidades da predominância da sífilis congênita nas regiões do Brasil. Revista Eletrônica Acervo Científico, 2020; 10: 1-8.

8. DOMINGUES CSB, et al. Protocolo Brasileiro para Infecções Sexualmente Transmissíveis 2020: sífilis congênita e criança exposta à sífilis. Epidemiol. Serv. Saúde, 2021; 30: 1-13.

9. FREITAS JLG, et al. Sífilis Materna e Congênita em Rondônia: casos notificados de 2010 a 2015. Revista Eletrônica Acervo Saúde, 2019; 11 (17): 1-9.

10. GRAY PB, et al. Age-Related Patterns in Sexual Behaviors and Attitudes Among Single U.S. Adults: An Evolutionary Approach. Evolutionary Behavioral Sciences. 2019; 13 (2), 111-126.

11. HOLZTRATTNER JS, et al. Sífilis congênita: realização do pré-natal e tratamento da gestante e de seu parceiro. Cogitare enferm. 2019; $24: 1-11$.

12. INSTITUTO BRASILEIRO DE GEOGRAFIA E ESTATÍSTICA (IBGE). Censo Demográfico 2010. Características gerais da população, religião e pessoas com deficiência. Rio de Janeiro: 2010; 215 p. Disponível em: https://biblioteca.ibge.gov.br/visualizacao/periodicos/94/cd_2010_religiao_deficiencia.pdf. Acessado em: 27/07/2021.

13. INSTITUTO BRASILEIRO DE GEOGRAFIA E ESTATíSTICA (IBGE). Diretoria de Pesquisas. Coordenação de Trabalho e Rendimento. Pesquisa Nacional por Amostra de Domicílios Contínua 2012-2019. Disponível em: https://agenciadenoticias.ibge.gov.br/media/com_mediaibge/arquivos/8ff41004968ad36306430c82eece3173.pdf. Acessado em: 27/07/2021. 
14. LAFETÁ KRG, et al. Sífilis materna e congênita, subnotificação e difícil controle. Rev Bras Epidemiol. 2016; 19(1): 6374.

15. LIMA VC, et al. Perfil epidemiológico dos casos de sífilis congênita em um município de médio porte no nordeste brasileiro. J. Health Biol Sci. 2017; 5(1): 56-61.

16. MAGALHÃES DMS, et al. Sífilis materna e congênita: ainda um desafio. Cad. Saúde Pública, 2013, 29(6):1109-1120.

17. MASCHIO-LIMA T, et al. Epidemiological profile of patients with congenital and gestational syphilis in a city in the State of São Paulo, Brazil. Revista Brasileira de Saúde Materno Infantil [online] 2019; 19(4): 873-880.

18. MINISTÉRIO DA SAÚDE. Protocolo Clínico e Diretrizes Terapêuticas para Prevenção da Transmissão Vertical do HIV, Sífilis e Hepatites Virais, 2019. Disponível em: http://www.aids.gov.br/pt-br/pub/2015/protocolo-clinico-e-diretrizesterapeuticas-para-prevencao-da-transmissao-vertical-de-hiv. Acessado em: 30/07/2021.

19. MINISTÉRIO DA SAÚDE. Secretaria de Atenção à Saúde. Departamento de Atenção Básica. Atenção ao pré-natal de baixo risco. Brasília: Editora do Ministério da Saúde, 2012 . Disponível em: https://bvsms.saude.gov.br/bvs/publicacoes/cadernos_atencao_basica_32_prenatal.pdf. Acessado em: 30/07/2021.

20. MINISTÉRIO DA SAÚDE. Secretaria de Vigilância em Saúde. Programa Nacional de DST, Aids e Hepatites Virais. Boletim Epidemiológico - Sífilis, 2018. Disponível em: http://www.aids.gov.br/pt-br/pub/2018/boletim-epidemiologicode-sifilis-2018. Acessado em: 21/07/2021.

21. MINISTÉRIO DA SAÚDE. Portaria o 542 de 22 de dezembro de 1986 . Disponível em: http://www3.crt.saude.sp.gov.br/arquivos/arquivos_biblioteca_crt/Portarian542de22dez86.pdf. Acessado em: 05/10/2021.

22. MINISTÉRIO DA SAÚDE. Portaria no 33 de 14 de julho de 2005 . Disponível em: https://bvsms.saude.gov.br/bvs/saudelegis/svs/2005/prt0033_14_07_2005.html\#: :text=Inclui\%20doen\%C3\%A7as\% 20\%C3\%A0\%20rela\%C3\%A7\%C3\%A30\%20de,de\%20Refer\%C3\%AAncia\%20Nacional\%20ou\%20Regional. Acessado em: 05/10/2021.

23. MINISTÉRIO DA SAÚDE. Portaria Ministerial ํำ104 de 25 de janeiro de 2011. Disponível em: https://bvsms.saude.gov.br/bvs/saudelegis/gm/2011/prt0104_25_01_2011.html. Acessado em: 05/10/2021.

24. MOREIRA KFA, et al. Perfil dos casos notificados de sífilis congênita. Cogitare Enferm., 2017; 22(2): 1-10.

25. MOTTA IA, et al. Sífilis congênita: por que sua prevalência continua tão alta? Revista Médica de Minas Gerais 2018; 28(6): 1-8.

26. SARACENI V, et al. Vigilância epidemiológica da transmissão vertical da sífilis: dados de seis unidades federativas no Brasil. Rev Panam Salud Publica, 2017; 41: 1-8.

27. SILVA LCVG, et al. Perfil dos casos de sífilis congênita em um município do sul de Mato Grosso. Journal Health NPEPS, 2017: 380-390.

28. SILVA LMC, et al. Sífilis congênita no estado do Pará-Brasil, 2007 a 2016. Revista Eletrônica Acervo Saúde, 2019; 24: 1-10.

29. SILVA MJN, et al. Distribuição da sífilis congênita no estado do Tocantins, 2007-2015. Epidemiol. Serv. Saude, Brasília, 2020; 29(2):1-8.

30. SOARES KKS, et al. Análise espacial da sífilis em gestantes e sífilis congênita no estado do Espírito Santo, 2011 2018. Epidemiol. Serv. Saude, 2020; 29(1:)1-12.

31. TEIXEIRA LO, et al. Tendência temporal e distribuição espacial da sífilis congênita no estado do Rio Grande do Sul entre 2001 e 2012. Ciência \& Saúde Coletiva. 2018; 23(8): 2587-97.

32. TOLDO MKS, et al. A recrudescência da sífilis congênita. Arquivos Catarinenses de Medicina, 2018; 47(1): 2-10. 